\title{
Evaluation of a Seton Procedure Combined With Infliximab Therapy (Early vs. Late) in Perianal Fistula With Crohn Disease
}

\author{
Myunghoon Jeon ${ }^{1}$, Kihwan Song ${ }^{1}$, Jail Koo ${ }^{1}$, Sohyun Kim ${ }^{2}$ \\ ${ }^{1}$ Department of Surgery, Goo Hospital, Daegu; ${ }^{2}$ Department of Surgery, Yeungnam University Medical Center, Yeungnam University College \\ of Medicine, Daegu, Korea
}

Purpose: We assessed the clinical outcomes of a seton procedure combined with early versus late institution of infliximab (IFX) therapy.

Methods: This retrospective study comprised 76 patients who underwent surgery for perianal fistula associated with Crohn disease between January 2014 and November 2017. All patients underwent loose seton drainage combined with IFX therapy. Patients categorized as the early group (EG, 49 patients) received IFX therapy within 30 days of completion of the seton procedure. Patients categorized as the late group (LG, 27 patients) received IFX therapy $>30$ days after the seton procedure. IFX therapy was administered as induction and maintenance therapy.

Results: There were no statistically significant intergroup differences in clinical characteristics of the patients. The mean follow-up was $21.0 \pm 11.6$ months in the EG and $34.5 \pm 18.4$ months in the LG $(P=0.001)$. The mean interval between seton procedure and IFX induction therapy was 12.2 days in the EG and 250.2 days in the LG $(\mathrm{P}=0.002)$. Complete remission was observed in 32 patients $(65.3 \%)$ in the EG and 17 patients $(63.0 \%)$ in the $\mathrm{LG}(\mathrm{P}=0.844)$. Fistula recurrence was observed in 6 patients (7.9\%). All recurrences occurred in a previous perianal fistula tract.

Conclusion: Patients showed a good response to a seton procedure combined with IFX therapy regardless of the time of initiation of IFX therapy.

Keywords: Crohn disease; Infliximab; Perianal fistula; Seton

\section{INTRODUCTION}

The incidence and prevalence of Crohn disease (CD) have increased rapidly in Asia, although these rates remain lower than those observed in Western countries $[1,2]$. However, Asian patients with CD typically have a more complicated and complex presentation than Western patients [1,2]. Notably, Asians with

Received: August 23, 2018 - Accepted: November 23, 2018

Correspondence to: Sohyun Kim, M.D.

Department of Surgery, Yeungnam University Medical Center, Yeungnam University College of Medicine, 170 Hyeonchung-ro, Nam-gu, Daegu 42415, Korea

Tel: +82-53-620-3594, Fax: +82-53-624-1213,E-mail: kshgs@yu.ac.kr ORCID code: https://orcid.org/0000-0002-8625-329X

(C) 2019 The Korean Society of Coloproctology

This is an open-access article distributed under the terms of the Creative Commons Attribution NonCommercial License (http://creativecommons.org/licenses/by-nc/4.0) which permits unrestricted noncommercial use, distribution, and reproduction in any medium, provided the original work is properly cited.
$\mathrm{CD}$ show higher frequency of stricture formation and penetrating disease than Australians [2], and $46.8 \%$ of Koreans with CD show perianal fistula formation before and after diagnosis [3].

Treatment of perianal fistulas associated with $\mathrm{CD}$ is difficult. Perianal fistulas of $\mathrm{CD}$ are complicated and may occasionally involve the large anal sphincter muscle [4]. These fistulas occasionally present as multiple fistulas, proctitis, anal strictures, abscesses, or rectovaginal fistulas [4-6]. Various treatments have been tried to treat perianal fistulas associated with $\mathrm{CD}$ [7-9]. A seton procedure is the most popular operation used to treat perianal fistulas in patients with $\mathrm{CD}$. The seton procedure reduces inflammation and allows adequate drainage of pus $[5,10]$; however, some patients must retain the seton over long periods of time $[10,11]$, which negatively affects their quality of life. Infusion of infliximab (IFX) is an established treatment for perianal fistulas in patients with CD $[11,12]$. Recently, a seton procedure combined with IFX therapy was reported to improve clinical outcomes in patients 
with $\mathrm{CD}$ who presented with perianal fistulas compared to conventional treatment [13].

Despite the proven efficacy of a seton procedure combined with IFX therapy, some patients choose not to receive IFX therapy immediately after a seton procedure owing to a variety of reasons, including poor infection control and socioeconomic issues. As a result, many patients may have already undergone the seton procedure before combined therapy using a seton procedure and IFX. The effect of delayed IFX therapy in these patients remains unclear, as few studies have demonstrated the clinical outcomes of a seton procedure combined with early or late IFX therapy. The aim of this study was to assess the clinical outcomes of a seton procedure combined with early vs. late IFX therapy.

\section{METHODS}

This retrospective study included patients who underwent surgery for treatment of perianal fistulas associated with $\mathrm{CD}$ between January 2014 and November 2017. All patients were diagnosed with complex anal fistulas due to $\mathrm{CD}$. Patients were diagnosed with $\mathrm{CD}$ by experienced gastrointestinal physicians, pathologists, or surgeons, and complex perianal fistulas were diagnosed by experienced specialist colorectal surgeons. A few patients were diagnosed with $\mathrm{CD}$ through histopathologic examination immediately after fistula surgery; these patients underwent laboratory, radiologic, and endoscopic examinations for $\mathrm{CD}$ postoperatively. Patients with typical symptoms of $\mathrm{CD}$ also underwent laboratory, radiologic, endoscopic, and histopathologic screening for diagnosis of CD. In some cases, diagnosis was delayed, and the aforementioned tests were repeated in these patients. All patients underwent loose seton drainage followed by IFX therapy. Patients were categorized into 2 groups: an early group (EG) and a late group (LG). Patients in the EG received IFX therapy within 30 days of completion of the seton procedure, whereas patients in the LG received IFX therapy $>30$ days after the seton procedure. This study received approval from the Institutional Review Board of Yeungnam University Medical Center (2018-08-012), who waved the need for informed consent as it was a retrospective study with minimal risk to patients.

All surgeries were performed by specialist colorectal surgeons. All patients underwent transanal ultrasonography, computed tomography of the abdominoperineal region, or pelvic magnetic resonance imaging (MRI) before anal surgery to assess type of fistula and/or presence of an abscess. All patients received prophylactic preoperative antibiotics (metronidazole or ciprofloxacin) and underwent spinal anesthesia and loose noncutting seton placement using a silicone band. The appropriate time for seton removal was determined by surgeons based on healing of the fistula tract, defined as reduction of inflammation and discharge, as well as reduced size of the fistula. Patients in the EG group showed improvement of their symptoms within one month of completion of the seton procedure. Eleven patients in the LG group had sustained inflammation and pus drainage for more than one month. The remaining 16 patients were treated with immunomodulator and antibiotics after the seton procedure, but there was no improvement of anal fistula.

Induction therapy with IFX was initiated after improvement in inflammation of the fistula and pus drainage. Laboratory and radiological tests were performed in all patients who received IFX therapy to confirm the absence of active infection or inactive tuberculosis prior to induction therapy. Induction therapy $(5 \mathrm{mg} /$ $\mathrm{kg}$ ) was at 0,2 , and 6 weeks. After induction therapy, maintenance therapy was performed every 8 weeks in patients who showed decreased anal discharge or a healing anal wound. No patient had been injected with IFX prior to this study.

Follow-up information was collected regularly at the time of IFX injection. The clinical response was documented and classified during outpatient visits. A partial response was defined as reduction of discharge from the fistula and/or size and number of fistulas. Complete remission was defined as complete closure of the fistula tract with cessation of drainage from the external opening of the fistula. Recurrence was defined as development of new fistulas or appearance of discharge from a former tract.

All statistical analyses were performed using IBM SPSS Statistics ver. 23.0 (IBM Co., Armonk, NY, USA) and R 3.5 with the rms and Hmisc packages (web-r.org). Continuous data were analyzed using Student t-test. Clinical characteristics and outcomes were analyzed using the chi-square or Fisher exact test.

\section{RESULTS}

We enrolled 76 patients in this study, 49 in the EG and 27 in the LG. CD was diagnosed at a mean age of 23.0 years (range, 10-52 years). Perianal fistulas were the first manifestation of disease in 48 patients (63.2\%). The overall mean duration of $\mathrm{CD}$ before a seton procedure was $13.0 \pm 2.9$ months. Among the 76 patients treated, 45 did not receive any medication for $\mathrm{CD}$ before they underwent the seton procedure combined with IFX therapy. The remaining patients received several medications for $\mathrm{CD}$. The followup period was longer in the $\mathrm{LG}$ than in the EG $(\mathrm{P}=0.001)$. Baseline characteristics of both groups at the time of fistula surgery are presented in Table 1. Medications used for CD at the time of the seton procedure are presented in Table 2 .

All patients received IFX therapy. The mean interval between the seton procedure and IFX induction therapy was 12.2 days (range, 2-27 days) in the EG and 250.2 days (range, 33-1,596 days $)$ in the LG $(P=0.002)$. All patients responded to IFX induction therapy. Of the 76 patients treated, $49(64.5 \%)$ showed complete remission after IFX induction therapy, 32 patients $(65.3 \%)$ in the EG and 17 patients $(63.0 \%)$ in the LG $(\mathrm{P}=0.844)$. Intergroup differences in the results of IFX induction therapy are presented in Table 3. Six patients (7.9\%) changed IFX therapy after induction; of these, 3 patients discontinued IFX therapy because of infusion reactions (one patient experienced pruritus, flushing, and 
Table 1. Clinical characteristics of the study patients

\begin{tabular}{lccc}
\hline Characteristic & EG $(n=49)$ & LG $(n=27)$ & P-value \\
\hline Sex & $12(24.5)$ & $9(33.3)$ & 0.577 \\
$\quad$ Female & $37(75.5)$ & $18(66.7)$ & \\
$\quad$ Male & $22.9 \pm 5.9$ & $26.6 \pm 10.5$ & 0.103 \\
Age (yr) & & & 0.326 \\
CD distribution & $5(10.2)$ & $2(7.4)$ & \\
Colon & $38(77.6)$ & $18(66.7)$ & \\
lleocecal & $1(2.0)$ & $3(11.1)$ & \\
Rectum & $5(10.2)$ & $4(14.8)$ & \\
SB & & & 0.224 \\
Fistula type & $19(38.8)$ & $6(22.2)$ & \\
Intersphincteric & $30(61.2)$ & $21(77.8)$ & \\
Transsphincteric & $13.3 \pm 25.1$ & $12.4 \pm 25.1$ & 0.886 \\
Mean duration of CD & & & 0.802 \\
Previous CD medication & $28(57.1)$ & $17(63.0)$ & \\
No & $21(42.9)$ & $10(37.0)$ & \\
Yes & $21.0 \pm 11.6$ & $34.5 \pm 18.4$ & 0.001 \\
\hline Follow-up period (mo) & & \\
\hline
\end{tabular}

Values are presented as number (\%) or mean \pm standard deviation. $E G$, early group; $L G$, late group; $C D$, Crohn disease.

skin rash; a second experienced pruritus, chest discomfort, nausea, and dizziness; and a third experienced flushing, nausea and urticarial), while 3 patients were switched to adalimumab because of activation of luminal CD. No significant difference in IFX maintenance therapy was evident $(\mathrm{P}=1.000)$.

Fistula recurrence was observed in 6 patients (7.9\%) at 5, 7, 8, 11,20 , or 22 months after initial IFX therapy. All recurrences occurred in a previous perianal fistula tract. A previous fistula tract reopened in 3 patients during IFX therapy; however, complete remission was observed after IFX induction therapy. Two patients experienced pus discharge during IFX therapy. One patient had fistula recurrence after switching to adalimumab because of a general inflammatory condition. No significant intergroup difference in fistula recurrence was evident $(\mathrm{P}=0.743)$ (Table 4$)$.

\section{DISCUSSION}

In this study, patients who underwent a seton procedure combined with IFX therapy had an acceptable response to this treatment. Complete remission of perianal disease was observed in $>60 \%$ of patients after IFX induction therapy, and $>88 \%$ of patients continued IFX maintenance therapy without severe complications and/or recurrence. No significant intergroup differences were found in the effects of IFX therapy based on timing of initiation of IFX therapy. A seton procedure combined with IFX therapy might improve treatment outcomes of perianal fistula regard-
Table 2. Medication at time of seton placement

\begin{tabular}{lc}
\hline Medication & No. of patients \\
\hline No & 45 \\
Mesalazine & $28^{\mathrm{a}}$ \\
Azathioprine & $16^{\mathrm{a}}$ \\
Steroid & $1^{\mathrm{a}}$ \\
Antibiotics & $4^{\mathrm{a}}$ \\
\hline
\end{tabular}

These figures include duplicate patients.

Table 3. Results of infliximab therapy

\begin{tabular}{lccc}
\hline Variable & $\mathrm{EG}(\mathrm{n}=49)$ & $\mathrm{LG}(\mathrm{n}=27)$ & P-value \\
\hline Induction therapy & & & 0.844 \\
$\mathrm{CR}$ & $32(65.3)$ & $17(63.0)$ & \\
PR & $17(34.7)$ & $10(37.0)$ & \\
Maintenance therapy & & & 1.000 \\
No & $4(8.2)$ & $2(7.4)$ & \\
Yes & $45(91.8)$ & $25(92.6)$ & \\
\hline
\end{tabular}

Values are presented as number (\%).

$E G$, early group; LG, late group; $C R$, complete remission; $P R$, partial response.

Table 4. Recurrence of perianal fistula

\begin{tabular}{lccc}
\hline & $\mathrm{EG}(\mathrm{n}=49)$ & $\mathrm{LG}(\mathrm{n}=27)$ & P-value \\
\hline Recurrence & & & 0.743 \\
No & $46(93.9)$ & $24(88.9)$ & \\
Yes & $3(6.1)$ & $3(11.1)$ & \\
\hline
\end{tabular}

Values are presented as number (\%).

$E G$, early group; $L G$, late group.

less of the timing of the seton procedure.

A seton procedure combined with anti-tumor necrosis factor (TNF) therapy is recommended for perianal fistulas associated with CD. The results of a seton procedure combined with IFX therapy have varied across previous studies. Complete remission of perianal fistulas occurred in $0 \%-78 \%$ of patients [13-15], and the overall response rate was approximately $71 \%-88 \%[14,16]$. In this study, the overall complete remission and response rates were $64.4 \%$ and $100 \%$, respectively. The findings in this study are within the range reported in previous studies.

This study reported $7.9 \%$ recurrence and $0 \%$ no-response rates. These figures are lower than the approximately $40 \%$ recurrence rate reported in previous studies $[14,17]$. The authors of these previous studies attributed the high recurrence rate to a short follow-up period. A few patients who showed initial response to IFX therapy developed worsening symptoms at approximately 41-50 months (during follow-up) $[18,19]$. The recurrence rates at 1,2 , and 3 years were reported to be $29.2 \%, 34.9 \%$, and $45.8 \%$, respectively [17], while the no/poor response rate was approximately 
28\%-37\% [9, 15]. In this study, 45 patients (59.2\%) had never received any medications for CD other than IFX therapy. Patients were simultaneously diagnosed with $\mathrm{CD}$ and perianal fistulas associated with CD. Patients received IFX therapy immediately after undergoing the seton procedure. The current strategy for management of CD involves "top-down" therapy with early institution of biological treatment [20]. The rates of mucosal healing were significantly higher in the "top-down" group than those in the conventional step-up group [21, 22]. The authors are of the view that the 45 patients might have benefitted from a top-down strategy.

Conventionally, combined anti-TNF- $\alpha$ antibody administration and seton procedure have been an important treatment strategy for perianal CD. IFX therapy is considered an important advance in the medical treatment of perianal $\mathrm{CD}$ and has shown a higher long-term response rate (approximately 56\%-68\%) than conventional therapy [12]. The ACCENT II study reported an approximately $36 \%$ response rate to IFX maintenance therapy at 54 weeks [23]. Patients who presented with complex perianal fistulas without anorectal sepsis had a $>90 \%$ response with IFX alone [13]. However, patients with perianal fistulas associated with CD frequently develop complex fistulas and anal sepsis before or after treatment $[13,14]$. Previous studies have shown that $>80 \%$ of patients who underwent a seton procedure presented with a complex fistula [14]. The rate of abscess formation after IFX therapy was reported to be approximately $11 \%-29 \%[12,19]$. Patients who underwent a loose seton technique showed $65 \%$ improvement in perianal sepsis at short-term follow-up and $20 \%$ improvement in perianal sepsis at long-term follow-up [11].

There are several limitations of this study. (1) Owing to the retrospective study design, we cannot exclude a possible selection bias related to choice of treatment based on clinical symptoms and (2) over $50 \%$ of the patients had never received medication for CD before receiving IFX therapy. Although a "top-down" approach with early administration of biological agents is considered useful to treat CD, IFX therapy in these patients may be controversial due to possible overtreatment [22]. In addition, (3) clinical response was determined based on clinical evaluation, and MRI or ultrasonography was not included in this study. In addition, our findings need to be confirmed by a multicenter controlled study.

In conclusion, we found a seton procedure combined with IFX therapy to be effective in patients presenting with perianal fistulas associated with $\mathrm{CD}$, and most patients showed better complete remission and response rates. Most patients were able to continue IFX therapy and showed low recurrence rates. The favorable response was achieved when combining a seton procedure and IFX therapy regardless of timing of initiation of IFX therapy.

\section{CONFLICT OF INTEREST}

No potential conflict of interest relevant to this article was reported.

\section{REFERENCES}

1. Prideaux L, Kamm MA, De Cruz PP, Chan FK, Ng SC. Inflammatory bowel disease in Asia: a systematic review. J Gastroenterol Hepatol 2012;27:1266-80.

2. Ng SC, Tang W, Ching JY, Wong M, Chow CM, Hui AJ, et al. Incidence and phenotype of inflammatory bowel disease based on results from the Asia-pacific Crohn's and colitis epidemiology study. Gastroenterology 2013;145:158-65.e2.

3. Ye BD, Yang SK, Cho YK, Park SH, Yang DH, Yoon SM, et al. Clinical features and long-term prognosis of Crohn's disease in Korea. Scand J Gastroenterol 2010;45:1178-85.

4. Lahat A, Assulin Y, Beer-Gabel M, Chowers Y. Endoscopic ultrasound for perianal Crohn's disease: disease and fistula characteristics, and impact on therapy. J Crohns Colitis 2012;6:311-6.

5. Sandborn WJ, Fazio VW, Feagan BG, Hanauer SB; American Gastroenterological Association Clinical Practice Committee. AGA technical review on perianal Crohn's disease. Gastroenterology 2003;125:1508-30.

6. Bergstrand O, Ewerth S, Hellers G, Holmström B, Ullman J, Wallberg P. Outcome following treatment of anal fistulae in Crohn's disease. Acta Chir Scand Suppl 1980;500:43-4.

7. Marzo M, Felice C, Pugliese D, Andrisani G, Mocci G, Armuzzi A, et al. Management of perianal fistulas in Crohn's disease: an upto-date review. World J Gastroenterol 2015;21:1394-403.

8. Chung W, Ko D, Sun C, Raval MJ, Brown CJ, Phang PT. Outcomes of anal fistula surgery in patients with inflammatory bowel disease. Am J Surg 2010;199:609-13.

9. El-Gazzaz G, Hull T, Church JM. Biological immunomodulators improve the healing rate in surgically treated perianal Crohn's fistulas. Colorectal Dis 2012;14:1217-23.

10. Thornton M, Solomon MJ. Long-term indwelling seton for complex anal fistulas in Crohn's disease. Dis Colon Rectum 2005;48: 459-63.

11. Buchanan GN, Owen HA, Torkington J, Lunniss PJ, Nicholls RJ, Cohen CR. Long-term outcome following loose-seton technique for external sphincter preservation in complex anal fistula. $\mathrm{Br} \mathrm{J}$ Surg 2004;91:476-80.

12. Present DH, Rutgeerts P, Targan S, Hanauer SB, Mayer L, van Hogezand RA, et al. Infliximab for the treatment of fistulas in patients with Crohn's disease. N Engl J Med 1999;340:1398-405.

13. Sciaudone G, Di Stazio C, Limongelli P, Guadagni I, Pellino G, Riegler G, et al. Treatment of complex perianal fistulas in Crohn disease: infliximab, surgery or combined approach. Can J Surg 2010;53:299-304.

14. Uchino M, Ikeuchi H, Bando T, Matsuoka H, Takesue Y, Takahashi $Y$, et al. Long-term efficacy of infliximab maintenance therapy for perianal Crohn's disease. World J Gastroenterol 2011;17: 1174-9.

15. Kotze PG, Albuquerque IC, da Luz Moreira A, Tonini WB, Olandoski M, Coy CS. Perianal complete remission with combined therapy (seton placement and anti-TNF agents) in Crohn's dis- 
ease: a Brazilian multicenter observational study. Arq Gastroenterol 2014;51:284-9.

16. Antakia R, Shorthouse AJ, Robinson K, Lobo AJ. Combined modality treatment for complex fistulating perianal Crohn's disease. Colorectal Dis 2013;15:210-6.

17. Haennig A, Staumont G, Lepage B, Faure P, Alric L, Buscail L, et al. The results of seton drainage combined with anti-TNFa therapy for anal fistula in Crohn's disease. Colorectal Dis 2015;17:3119.

18. Duff S, Sagar PM, Rao M, Dolling S, Sprakes M, Hamlin PJ. Infliximab and surgical treatment of complex anal Crohn's disease. Colorectal Dis 2012;14:972-6.

19. Bouguen G, Siproudhis L, Gizard E, Wallenhorst T, Billioud V, Bretagne JF, et al. Long-term outcome of perianal fistulizing Crohn's disease treated with infliximab. Clin Gastroenterol Hepatol 2013;11:975-81.e1-4.
20. Hanauer SB. Clinical perspectives in Crohn's disease. Turning traditional treatment strategies on their heads: current evidence for "step-up" versus “top-down". Rev Gastroenterol Disord 2007;7 Suppl 2:S17-22.

21. Baert F, Moortgat L, Van Assche G, Caenepeel P, Vergauwe P, De Vos M, et al. Mucosal healing predicts sustained clinical remission in patients with early-stage Crohn's disease. Gastroenterology 2010;138:463-8.

22. D'Haens G, Baert F, van Assche G, Caenepeel P, Vergauwe P, Tuynman H, et al. Early combined immunosuppression or conventional management in patients with newly diagnosed Crohn's disease: an open randomised trial. Lancet 2008;371:660-7.

23. Sands BE, Anderson FH, Bernstein CN, Chey WY, Feagan BG, Fedorak RN, et al. Infliximab maintenance therapy for fistulizing Crohn's disease. N Engl J Med 2004;350:876-85. 